\title{
Metodología de análisis de forjados autotesados de madera
}

\section{Methodology for the analysis of self-tensioned wooden structural floors}

\author{
$\underline{\text { F. Suárez-Riestra }}^{(*)}$, J. Estévez-Cimadevila ${ }^{(*)}$, D. Otero-Chans ${ }^{(*)}$
}

\section{RESUMEN}

Se describe un sistema de autotesado constituido por un dispositivo multiplicador de fuerza que acoplado a los apoyos extremos del elemento estructural resulta capaz de convertir la resultante vertical de las acciones gravitatorias en una acción efectiva de tesado mediante el movimiento inducido en un juego de bielas. El sistema de autotesado es capaz de ofrecer unas altas prestaciones gracias al efecto beneficioso de la deformación contraria generada por el tesado de forma proporcional al incremento de la acción gravitatoria. Ello permite diseñar forjados nervados de madera de elevada luz empleando cantos reducidos.

La complejidad de cálculo debida a la no linealidad del sistema se puede obviar con la metodología de análisis desarrollada en el artículo.

Con objeto de ilustrar las ventajas del sistema de autotesado y de la metodología de análisis desarrollados se han analizado seis casos de forjado nervado con luces de 9, 12 y $15 \mathrm{~m}$ y cargas variables de uso de 3,00 kN/m² y 5,oo kN/m².

Palabras clave: pretensado; autotesado; forjados de grandes luces; forjados de madera; construcción en madera.

\section{ABSTRACT}

It is described a self-tensioning system constituted by a force multiplying device which, attached to the supports of the ends of the structural element, is able to convert the vertical resultant from the gravitatonial actions into an effective tensioning action, through the movement that was induced by a set of rods. The self-tensioning system is able to offer a high performance, thanks to the beneficial effect of the opposite deflection generated by the tensioning, in proportion to the increasing of the gravitational action. This allows to design long-span timber ribbed floors using reduced depths.

The complexity of calculation due to the non-linearity of the system can be obviated with the methodology of analysis developed in the article.

In order to illustrate the advantages of the self-tensioning system and the methodology of analysis which were developed, six cases of ribbed floors have been analysed, with spans of 9, 12 and $15 \mathrm{~m}$ and variable using loads of $3,00 \mathrm{kN} / \mathrm{m}^{2}$ and $5,00 \mathrm{kN} / \mathrm{m}^{2}$.

Keywords: pre-stressing; self-tensioning; long-span flooring; timber flooring; timber construction.

(*) Departamento Tecnología de la Construcción. Universidade da Coruña (España).

Persona de contacto/Corresponding author: felix.suarez@udc.es (F. Suárez-Riestra)

ORCID: http://orcid.org/oooo-0002-8839-5611 (F. Suárez-Riestra); http://orcid.org/oooo-0002-8460-2097

(J. Estévez-Cimadevila); http://orcid.org/oooo-0oo3-1738-252X (D. Otero-Chans)

Cómo citar este artículo/Citation: Suárez-Riestra, F., Estévez-Cimadevila, J., Otero-Chans, D. (2017). Metodología de análisis de forjados autotesados de madera. Informes de la Construcción, 69(547): e207, doi: http://dx.doi.org/10.3989/ic.16.076.

Copyright: (C) 2017 CSIC. Licencia / License: Salvo indicación contraria, todos los contenidos de la edición electrónica de Informes de la Construcción se distribuyen bajo una licencia de uso y distribución Creative Commons Attribution License (CC BY) Spain 3.o. 


\section{INTRODUCCIÓN}

El proceso de análisis de un forjado de gran luz resuelto en madera conduce habitualmente a un dimensionado derivado del cumplimiento de las exigencias de aptitud al servicio. Estas exigencias están recogidas normativamente de manera genérica mediante el establecimiento de unos determinados límites de deformación comunes para cualquier tipo estructural. En el caso de la madera la deformación diferida o de fluencia incrementa de forma significativa las deformaciones instantáneas, lo que hace que el cumplimiento de los límites de deformación obligue al empleo de secciones de dimensión importante, poco esbeltas y claramente sobredimensionadas desde el punto de vista tensional.

En el caso particular de los forjados de piso la presencia de una contraflecha inicial puede resultar efectiva en algunos casos de combinación de acciones, especialmente en el caso de cubiertas con una carga variable de reducida magnitud. Para mayores valores de carga variable la solución de contraflecha geométrica inicial es insuficiente y el cumplimiento de ELS, en el caso de integridad de elementos constructivos, sigue exigiendo el empleo de secciones robustas sobredimensionadas a nivel tensional.

Una alternativa eficaz para el control de deformaciones lo constituye la implementación de un mecanismo de pretensado, en tanto que su efecto conduce necesariamente a una deformación inversa a la generada por las acciones gravitatorias. Sin embargo, esta contraflecha inducida resulta incapaz de ofrecer soluciones válidas para situaciones en las que la relación entre la carga de carácter permanente $(G)$ y la de carácter variable $(Q)$ es baja. En los sistemas estructurales de madera el valor de la carga permanente resulta reducido frente a la variable determinada por el uso, especialmente cuando consideramos el uso público al que habitualmente se destinan los sistemas que han de salvar grandes luces.

Provocar un pretensado inicial que contemple la situación de contrarresto de la deformación debida a una determinada combinación de acciones puede conducir a una deformación excesiva en la situación de presencia exclusiva de carga permanente. Por otra parte, al ser significativa la magnitud de la carga variable, el cumplimiento de las limitaciones de deformación por integridad de elementos constructivos conduce igualmente a secciones de elevada inercia, por lo que la solución pretensada sigue sin proporcionar una solución eficiente del problema.

El sistema planteado da lugar a un comportamiento no lineal del elemento estructural, de manera que resulta complejo abordar una metodología sencilla que permita el análisis y diseño de estos sistemas. En el presente documento se establecen cuáles son los factores de los que depende la eficacia del sistema y su interrelación, proponiendo una formulación explícita que permite el estudio del comportamiento del sistema en servicio, prescindiendo de la complejidad analítica asociada a la no linealidad.

\section{SISTEMA DE AUTOTESADO}

Frente a estas alternativas el Grupo de Estructuras Arquitectónicas (GEA) de la Universidad de A Coruña (UDC), al amparo del Proyecto de Investigación «Sistemas prefabricados de altas prestaciones en madera laminada pretensada con tendones no adherentes» ha desarrollado un mecanismo denominado $\mathrm{SsS}^{\circledR}$ capaz de generar una fuerza de tesado variable de acuerdo a la acción gravitatoria que actúa en cada instante sobre el sistema estructural. Este mecanismo de autotesado acoplado a ambos apoyos extremos de la viga convierte la resultante vertical de las acciones gravitatorias $\left(F_{i}\right)$ en una acción horizontal $\left(N_{\text {post } i}\right)$ que dada la excentricidad de su punto de aplicación origina una acción efectiva de tesado. Esta conversión de acción exterior de componente vertical en acción interior de respuesta horizontal es posible mediante el movimiento inducido en un juego de bielas (Figuras 1 y 2).

Este sistema de bielas, debido a su configuración geométrica, es capaz de provocar un efecto multiplicador, amplificando la componente horizontal que actúa sobre el tensor generada por la acción vertical que recibe el juego de bielas (Figura 2).

El sistema de autotesado es capaz de ofrecer unas altas prestaciones, aportando el efecto beneficioso de una deformación contraria generada por el tesado de forma proporcional al incremento de la acción gravitatoria. El efecto positivo del mecanismo de autotesado y la incidencia de los diferentes parámetros de diseño han sido ya analizados para diferentes consideraciones de luz y carga (1) (2).

\section{METODOLOGÍA DE ANÁLISIS}

El sistema de autotesado se fundamenta en la presencia de fuerzas cambiantes relacionadas con geometrías dependientes, lo que conduce a una no linealidad geométrica ligada a esa autointeracción. En consecuencia, el análisis del comportamiento de este sistema no puede ser afrontado desde suposiciones matemáticas directas o desde simples aproximaciones que permitan obtener una solución a partir del principio de superposición.

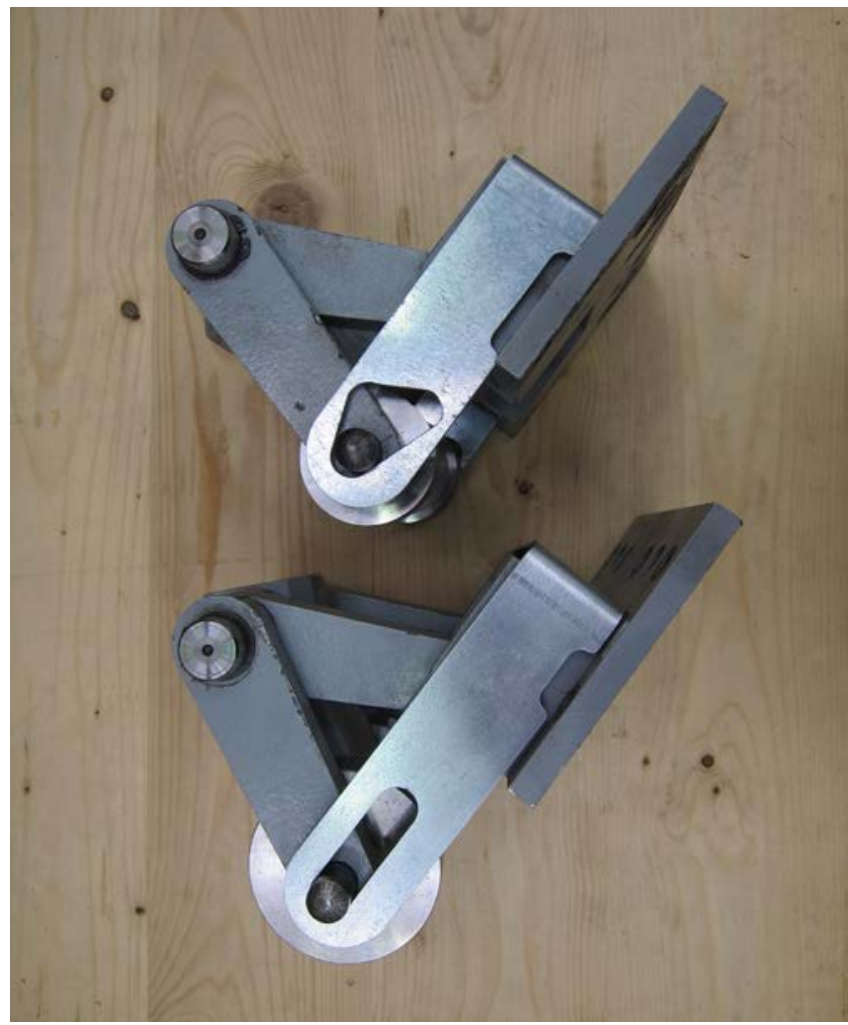

Figura 1. Imagen del mecanismo de autotesado $\left(\mathrm{SsS}^{\circledR}\right)$ en diferentes configuraciones geométricas. 


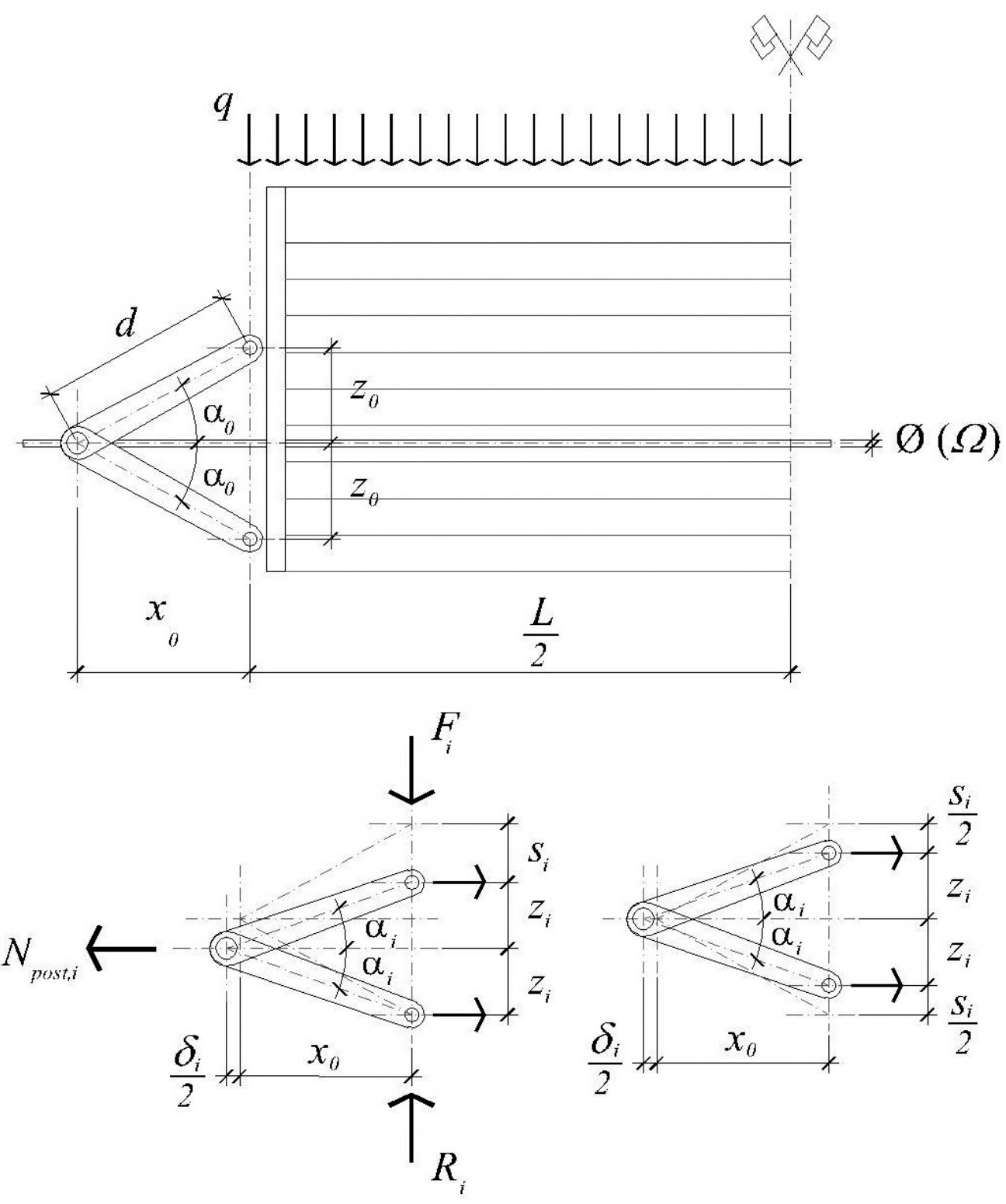

Figura 2. Esquema de trabajo del mecanismo de autotesado ( $\left.\mathrm{SsS}^{\circledR}\right)$.

La no linealidad geométrica que caracteriza el comportamiento del sistema imposibilita el cálculo de la situación deformada final de la pieza en un solo paso, ni siquiera en un proceso iterativo. Es posible realizar una aproximación a este comportamiento no lineal mediante un proceso incremental de carga, determinando la respuesta del sistema para cada uno de esos incrementos. Sin embargo, esta metodología exige además establecer un parámetro al cual referir el propio proceso incremental de carga. El análisis adopta así un valor de la variable que se desconoce y se modifica a medida que se introducen las exigencias que dicha variable debe verificar.

Cualquier método de resolución de un sistema de no linealidad geométrica combinará el proceso incremental de aplicación de cargas con un proceso iterativo dentro de cada paso de carga, que se encarga de buscar el equilibrio al final de dicho paso de carga (3). Esta condición exige una predicción previa del incremento de deformación que producirá el aumento de carga que, necesariamente, conducirá a una situación de desequilibrio, lo que obligará a un segundo proceso de corrección de la deformación. Estos procesos de corrección a los que atienden propuestas como el Método Incremental
Puro o el Método de Newton-Raphson (4) resultan muy efectivos en sistemas en los que la respuesta resulta creciente en orden a un proceso progresivo de incremento de carga. Pero en situaciones en las que el sistema puede presentar alteraciones en esta respuesta, por ejemplo con saltos bruscos en el valor de la deformación frente a pequeños incrementos de carga o incluso a fenómenos de inversión en la deformación, resulta aconsejable el empleo de Métodos Restringidos. Estos métodos de análisis contemplan la posibilidad de trabajar con funciones de varias variables que interesa maximizar o minimizar, estando sujetas a determinadas restricciones. Resulta indicado para sistemas en las que no se presenta un tipo de respuesta monótona frente a un proceso de carga incremental y básicamente se fundamenta en modificar el nivel de carga aplicada en cada paso del proceso incremental de carga en vez de mantenerlo constante (3).

En nuestro caso la presencia de una variación geométrica del sistema provoca una modificación de las acciones presentes, en tanto que el efecto del multiplicador induce para cada situación geométrica una acción diferente. La consideración de un Método Restringido permite modificar el nivel de carga 
aplicada en cada paso del proceso, en lugar de mantenerlo constante. Es decir, el nivel de carga resulta una variable más del problema que se autocondiciona a la deformación del sistema, de tal manera que en lugar de abordar un proceso incremental de la acción se introduce un algoritmo de control de la deformación.

Si bien esta metodología permite un estudio de las condiciones de trabajo del sistema, no resulta un medio idóneo para abordar un proceso de diseño o dimensionado del mismo. Sin embargo, la definición o diseño del sistema puede ser desarrollado si se establecen algunas restricciones precisas sobre el objetivo final de este dimensionado. Si se asume que en forjados de luz importante dotados del sistema de autotesado la condición restrictiva de dimensionado es el cumplimiento de ELS y, concretamente, de deformación, las variables acotadas han de ser aquellas que determinan dicha deformación.

En condición de trabajo el sistema experimentará dos tipos de deformación vertical de los que surgirá el valor de la distorsión resultante en el conjunto. En un instante cualquiera ( $i$ ) la presencia de una acción exterior $\left(q_{i}\right)$ provocará una resultante en cada extremo $\left(F_{i}\right)$ que determina el descenso vertical o asiento del apoyo $\left(s_{i}\right)$ (Figura 2). La modificación de geometría de este elemento conduce a su vez a una deformación en la dirección horizontal $\left(\delta_{i} / 2\right)$ que provoca un axil que conduce al tesado del tendón $\left(N_{\text {post.i }}\right)$. Las dos acciones presentes, la exterior y la interior de tesado, provocan un estado de flexión compuesta que provoca una deformación vertical cuyo valor máximo $\left(u_{i}\right)$ se situará en el punto medio de la pieza.

$\mathrm{Al}$ objeto de clarificar la nomenclatura utilizada se han representado con $(s)$ los asientos de los apoyos, con $(u)$ las flechas máximas, entendidas como el desplazamiento vertical de la sección central del elemento a partir de una situación dada, debido a la actuación de una acción cualquiera; con $(w)$ las flechas relativas o distorsión, entendida como el desplazamiento de la sección central del elemento medido respecto a la posición de los apoyos (Figura 3).

Para un instante de carga $\left(q_{i}\right)$ es posible evaluar la deformación vertical instantánea $\left(u_{i n s t i}\right)$ que resulta a partir de las expresiones conocidas de la Resistencia de Materiales. Para ello y adoptando un factor de rigidez a flexión $(\varphi)$ función de la luz $L$ y de las características geométricas (momento de inercia de la sección de madera, $I_{\text {beam }}$ ) y materiales (módulo de elasticidad de la madera, $E_{\text {beam }}$ ) del elemento en análisis, resultará:

$$
\varphi=8 \cdot \frac{E_{\text {beam }} \cdot I_{\text {beam }}}{L}
$$

$$
u_{i n s t, i}=\frac{L}{\varphi} \cdot\left(\frac{5}{6} \cdot M_{i s o s t, i} \cdot L^{2}-N_{p o s t, i} \cdot e\right)
$$

Esta expresión no es determinante, ya que en ella se presenta el axil de tesado cuyo resultado depende de la modificación geométrica del mecanismo de transferencia, y en concreto del descenso vertical $\left(s_{i}\right)$. Se produce, por tanto, una relación condicionada, en tanto que es el asiento producido en cada instante el que determinará la distorsión resultante $\left(\omega_{\text {inst }, i}\right)$.

Establecidos los factores que determinan la función objetivo podemos identificar los diferentes parámetros que intervienen en el análisis. Para ello y adoptando la propuesta del Método Restringido estableceremos aquellos parámetros que nos permitan controlar en todo momento el valor de la acción resultante del tensor en función de la variación geométrica del sistema encargado de trasladar dicha acción.

De la invariabilidad geométrica de los elementos que conforman el mecanismo multiplicador es posible establecer la consideración siguiente que se verifica en cualquier instante de carga (Figura 2).

$$
d^{2}=x_{\mathrm{o}}^{2}+z_{\mathrm{o}}^{2}=\left(x_{\mathrm{o}}+\frac{\delta_{i}}{2}\right)^{2}+\left(z_{\mathrm{o}}+\frac{s_{i}}{2}\right)^{2}
$$

Expresión que nos puede conducir a la determinación del alargamiento que experimentará el tensor $\left(\delta_{i}\right)$ en función de la geometría original del mecanismo y del asiento $\left(s_{i}\right)$ experimentado en un momento dado, eliminando de esta manera la componente de acción $\left(q_{i}\right)$ que lo provoca.

$$
\delta_{i}=\sqrt{s_{i} \cdot\left(4 \cdot z_{\mathrm{o}}-s_{i}\right)+4 \cdot x_{\mathrm{o}}^{2}}-2 \cdot x_{\mathrm{o}}
$$

Si definimos un nuevo factor de asiento $\left(\xi_{i}\right)$ es posible trascribir la expresión anterior en otra más simple en la que de nuevo se opera exclusivamente con relaciones geométricas.

$$
\begin{gathered}
\xi_{i}=\frac{s_{i}}{2}-z_{\mathrm{o}} \\
\delta_{i}=2 \cdot\left[\sqrt{d^{2}-\xi_{i}^{2}}-x_{\mathrm{o}}\right]
\end{gathered}
$$

Del equilibrio de fuerzas que se produce con el multiplicador es posible determinar el axil de tesado $\left(N_{\text {post }, i}\right)$ en un instante dado a partir de la geometría a la que conduce la presencia de una acción exterior $\left(q_{i}\right)$, de tal manera que:

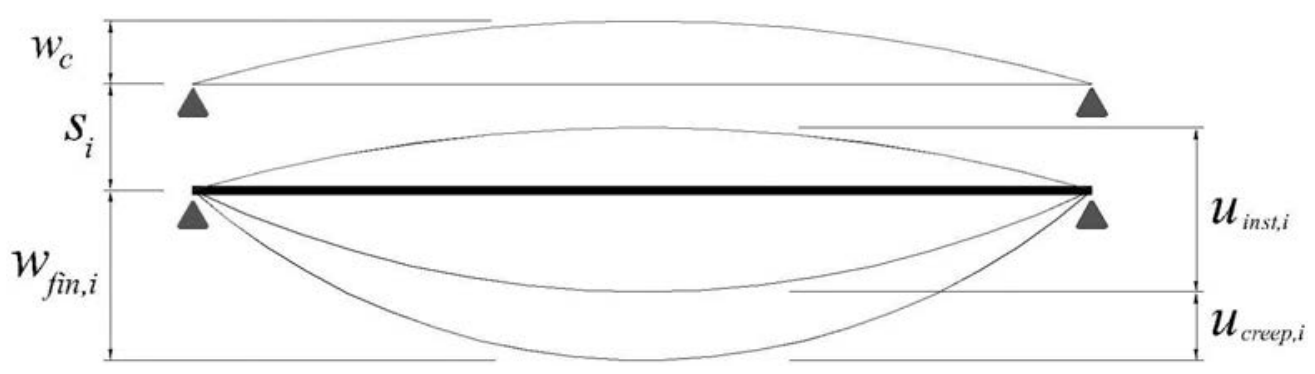

Figura 3. Nomenclatura empleada en el análisis de deformaciones. 


$$
\begin{gathered}
N_{p o s t, i}=\frac{2}{\operatorname{tg} \alpha_{i}} \cdot\left(\frac{q_{i} \cdot L}{2}\right) \\
\operatorname{tg} \alpha_{i}=\frac{z_{\mathrm{o}}-\frac{s_{i}}{2}}{x_{\mathrm{o}}+\frac{\delta_{i}}{2}} \\
N_{p o s t, i}=q_{i} \cdot L \cdot \frac{2 \cdot x_{\mathrm{o}}+\delta_{i}}{2 \cdot z_{\mathrm{o}}-s_{i}}
\end{gathered}
$$

Por otro lado es posible determinar el alargamiento que experimentará un tensor sometido a este axil a partir de su área $(\Omega)$ y del módulo de elasticidad $\left(E_{\text {tendon }}\right)$ del material constituyente.

$$
\delta_{i}=N_{\text {post }, i} \cdot \frac{L}{\Omega \cdot E_{\text {tendon }}}
$$

Definiendo un factor de rigidez axil $(\kappa)$ para el tendón de tesado y trasladando a la expresión las relaciones geométricas que conducen al ángulo resultante $\left(\alpha_{i}\right)$ en el instante de aplicación de la carga, podemos obtener una nueva expresión.

$$
\begin{gathered}
\kappa=\frac{\Omega \cdot E_{\text {tendon }}}{L} \\
\delta_{i}=\frac{q_{i} \cdot L}{\kappa} \cdot \frac{2 \cdot x_{\mathrm{o}}+\delta_{i}}{2 \cdot z_{\mathrm{o}}-s_{i}}=\frac{q_{i} \cdot L}{\kappa} \cdot\left(\frac{2 \cdot x_{\mathrm{o}}+\delta_{i}}{-2 \cdot \xi_{i}}\right)
\end{gathered}
$$

Es posible interrelacionar los parámetros determinantes, de tal manera que prefijado uno de ellos resulten condicionados el resto. De las expresiones [6] y [9] se deduce la extrema sensibilidad de la razón geométrica del multiplicador en el comportamiento del sistema, cuestión que resulta evidente, pero que permite así ser evaluada. Esta situación conduce a la determinación del parámetro de asiento $\left(s_{i}\right)$ que experimenta el apoyo como el valor determinante que permite el control del proceso de diseño del sistema. En base a este parámetro se deducen el resto de los parámetros geométricos que establecen la situación de equilibrio entre la acción exterior y la acción interior que la acompaña.

Las expresiones anteriores permiten establecer el comportamiento en un instante cualquiera (i) sin más que predeterminar el comportamiento en un instante previo de diseño (D) a partir de la definición del asiento pretendido en dicho instante previo. En el instante $D$, en el que actúa una carga $q_{D}$, de acuerdo a las expresiones determinadas, resultan los siguientes valores de axil de tesado y alargamiento del tensor:

$$
\begin{gathered}
N_{p o s t, D}=q_{D} \cdot L \cdot \frac{2 \cdot x_{\mathrm{o}}+\delta_{D}}{2 \cdot z_{\mathrm{o}}-s_{D}} \\
\delta_{D}=\frac{q_{D} \cdot L}{\kappa} \cdot \frac{2 \cdot x_{\mathrm{o}}+\delta_{D}}{2 \cdot z_{\mathrm{o}}-s_{D}}
\end{gathered}
$$

Agrupando términos en las expresiones [9], [10], [13] y [14] es posible alcanzar la siguiente igualdad:

$$
\frac{\delta_{D}}{q_{D}} \cdot \frac{2 \cdot z_{\mathrm{o}}-s_{D}}{2 \cdot x_{\mathrm{o}}+\delta_{D}}=\frac{\delta_{i}}{q_{i}} \cdot \frac{2 \cdot z_{\mathrm{o}}-s_{i}}{2 \cdot x_{\mathrm{o}}+\delta_{i}}
$$

Determinando un factor de comportamiento $(\eta)$ que tiene en cuenta la geometría resultante en el estadio de diseño $(D)$ y la razón entre las cargas actuantes en ambos instantes y aplicando el factor de asiento $\left(\xi_{i}\right)$ definido en [5] la anterior expresión adopta una nueva forma.

$$
\begin{gathered}
\eta_{i}=\frac{q_{i}}{q_{D}} \cdot\left[\delta_{D} \cdot \frac{2 \cdot z_{\mathrm{o}}-s_{D}}{2 \cdot x_{\mathrm{o}}+\delta_{D}}\right] \\
\eta_{i}+2 \cdot \xi_{i}=\frac{2 \cdot x_{\mathrm{o}} \cdot \xi_{i}}{\sqrt{d^{2}-\xi_{i}^{2}}}
\end{gathered}
$$

La resolución de esta ecuación conduce a un polinomio de cuarto grado que adopta la forma siguiente.

$$
\xi_{i}^{4}+\eta_{i} \cdot \xi_{i}^{3}-\left[z_{\mathrm{o}}^{2}-\left(\frac{\eta_{i}}{2}\right)^{2}\right] \cdot \xi_{i}^{2}-\eta_{i} \cdot d^{2} \cdot \xi_{i}-\left[d^{2}-\left(\frac{\eta_{i}}{2}\right)^{2}\right]=0
$$

La resolución de este polinomio conduce a cuatro raíces, de las cuales la que determina el valor mínimo representa la solución que responde al comportamiento del sistema. De esta manera, determinado el valor del factor de asiento $\left(\xi_{i}\right)$ correspondiente al instante $(i)$ es posible establecer el correspondiente asiento en dicho instante $\left(s_{i}\right)$, lo que permite obtener el resto de valores determinantes del resultado final.

De las expresiones anteriormente desarrolladas resulta posible determinar el proceso de respuesta en valores de acción y deformación que experimentará el sistema bajo cualquier situación de carga exterior. Para ello no resulta necesario más que establecer el asiento inicial en el apoyo $\left(s_{D}\right)$ como parámetro determinante de diseño, pudiendo obtener resultados de análisis del sistema que responde a una no linealidad geométrica obviando todo el proceso iterativo.

Esta consideración permite además una ventaja adicional al posibilitar el análisis de la deformación que resulta en un instante cualquiera $\left(u_{i}\right)$. Para poder aplicar las diferentes consideraciones que sobre deformación de sistemas estructurales se establecen en el punto A.1.4.3 de la UNE-EN 1990:2003 (5) se desarrolla una formulación que permite determinar la flecha instantánea que se produce bajo cualquier combinación de carga. Se establece un factor $\beta_{i}=Q_{i} / G$, que relaciona el valor de la carga de carácter permanente $(G)$ y el de la carga variable $\left(Q_{i}\right)$ en el instante (i) en el que queremos conocer la deformación, de tal manera que la carga permanente, por su carácter, se convierte en valor determinante. La formulación resultante permite integrar este conjunto de variables mediante la siguiente expresión:

$$
\omega_{\text {inst }, i}=\frac{M_{G, \text { isost }}}{\varphi}(1+\beta)\left(\frac{5 \cdot L}{6}-8 \cdot e \cdot \frac{2 \cdot x_{0}+\delta_{i}}{2 \cdot z_{0}-s_{i}}\right)-\omega_{c}
$$

Surge de esta manera un criterio de análisis que permite establecer las condiciones de dimensionado de las partes integrantes del sistema a partir de una sección geométrica determinada, de las propiedades mecánicas de los materiales constituyentes y de los parámetros de deformación pretendidos para cada instante de servicio. 


\section{APLICACIÓN DEL MÉTODO DE ANÁLISIS}

Como ya se ha indicado, el parámetro determinante en el proceso de diseño lo constituye el descenso experimentado en el apoyo en el instante $D\left(s_{D}\right)$. Prefijar un valor de $s_{D}$, para una determinada condición geométrica y material de la sección transversal y dada una situación prevista de carga, permite establecer el resto de factores variables que intervienen (alargamiento tendón, área precisa del tendón, fuerza de tesado, deformación instantánea) de acuerdo con:

$$
\begin{gathered}
\xi_{D}=\frac{s_{D}}{2}-z_{\mathrm{o}} \\
\delta_{D}=2 \cdot\left[\sqrt{d^{2}-\xi_{D}^{2}}-x_{\mathrm{o}}\right] \\
\Omega=\frac{q_{D} \cdot L}{E_{\text {tendon }} \cdot \delta_{D}} \cdot\left(\frac{2 \cdot x_{\mathrm{o}}+\delta_{D}}{2 \cdot z_{\mathrm{o}}-s_{D}}\right) \\
N_{\text {post }, D}=q_{D} \cdot L \cdot \frac{2 \cdot x_{\mathrm{o}}+\delta_{D}}{2 \cdot z_{\mathrm{o}}-s_{D}} \\
u_{\text {inst }, D}=\frac{L}{\varphi} \cdot\left(\frac{5}{6} \cdot M_{q_{D}, i \text { sost }} \cdot L^{2}-N_{p o s t, D} \cdot e\right)
\end{gathered}
$$

Atendiendo a estas consideraciones y a una condición de trabajo adecuada al proceso edificatorio al que se destina la propuesta, se establece un criterio que permite la optimización de la solución.

Si bien se puede fijar como criterio de diseño la consideración de cualquier hipótesis combinatoria de carga, una opción razonable, especialmente en el caso de cargas variables medias, es considerar la hipótesis combinatoria cuasipermanente. Dicha hipótesis de carga corresponde a la situación habitual de servicio de una estructura. Además, el cumplimiento de la exigencia normativa de la limitación de deformación por apariencia debe verificarse para la combinación cuasipermanente.

La utilización de esta hipótesis como la referencia de diseño permite, fijando un asiento en el apoyo $s_{D}$, calcular la flecha final (instantánea y diferida) y determinar la contraflecha de fabricación $\left(\omega_{c}\right)$ de forma que se obtenga una flecha relativa o distorsión nula, permaneciendo la pieza en horizontal para dicha hipótesis de carga.

A partir de esta situación de diseño, la deformada de la pieza para cualquier otro tipo de hipótesis combinatoria (i) se puede determinar resolviendo el polinomio [18], que proporciona el factor de asiento $\left(\xi_{i}\right)$, y aplicando las expresiones [5], [6], [9] y [19]. La metodología de análisis desarrollada aporta así una doble ventaja: por una parte, la sencillez de cálculo que permite solventar de forma extremadamente sencilla la problemática del análisis no lineal, que exigiría un proceso incremental en la aplicación de cargas combinado con un proceso iterativo en cada fase de carga; las expresiones desarrolladas se pueden implementar en una simple hoja de cálculo. En segundo lugar, la metodología de análisis permite evaluar de forma instantánea la incidencia de todos los parámetros de diseño que caracterizan el comportamiento del sistema. Se convierte, por tanto, en una potente herramienta de diseño del sistema pues, fijado el asiento en una situación concreta de carga, se determinan automáticamente los restantes factores (área del tendón, contraflecha) con los que se asegura la consecución del objetivo de diseño (usualmente, una distorsión nula en la hipótesis cuasipermanente de carga).

Con objeto de mostrar tanto la eficiencia del sistema de autotesado como de la metodología de análisis desarrollados, se acompañan los resultados correspondientes a unos forjados nervados con las siguientes características (Tabla 1).

- Luces de 9, 12 y 15 m.

- Carga permanente $G_{k}$ de $1 \mathrm{kN} / \mathrm{m}^{2}$ además del peso propio del elemento. Se han considerado dos situaciones para la determinación de la carga variable de uso: uso administrativo $\left(Q_{k}=3 \mathrm{kN} / \mathrm{m}^{2}, \psi_{2}=0,3\right)$ y uso de pública concurrencia $\left(Q_{k}=5 \mathrm{kN} / \mathrm{m}^{2}, \psi_{2}=0,6\right)$.

- Sección transversal tipo «T» conformada por un nervio de madera laminada, clase resistente GL28h (6) y tablero superior de madera contralaminada formado por 3 láminas o capas C24 (7). Para la determinación de las dimensiones de la sección transversal se ha utilizado una esbeltez $H=0,030 L$ para el caso de la carga variable de $3 \mathrm{kN} / \mathrm{m}^{2}$ y $H=0,033 L$ para $5 \mathrm{kN} / \mathrm{m}^{2}$, de acuerdo con los resultados obtenidos en el estudio de la incidencia de los distintos parámetros de diseño (1) (2).

- La geometría específica del dispositivo de autotesado y los valores de asiento de diseño se recogen en las Tablas 1 y 2

Tabla 1. Características formales y materiales de los forjados nervados mostrados.

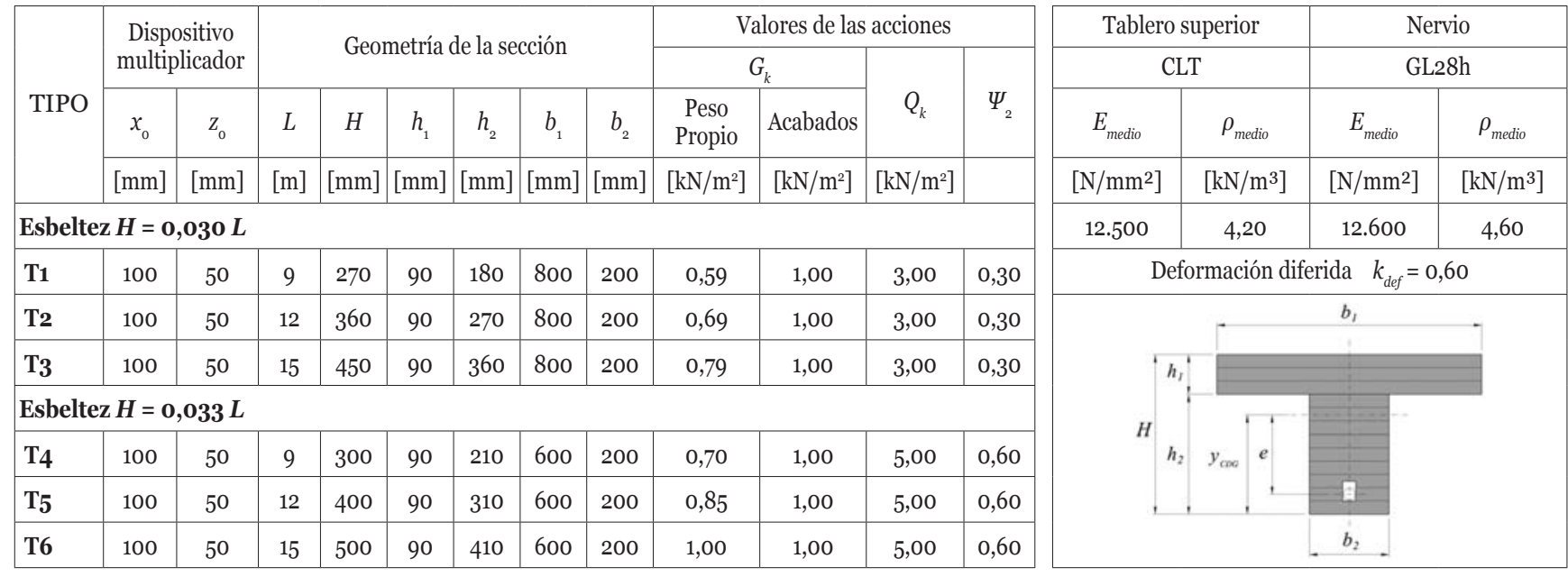


y han sido escogidos de forma que se obtenga una elevada eficiencia del sistema y el cumplimiento de las exigencias normativas en cuanto a deformaciones.

Para el cálculo se ha considerado cuatro hipótesis:

\section{- Hipótesis D.}

Se utiliza como hipótesis de diseño la correspondiente a la actuación de una acción exterior $\left(q_{D}\right)$ dada por:

$$
q_{D}=\sum_{j \geq 1} G_{k, j}+\sum_{i \geq 1} 0,3 \cdot Q_{k, i}
$$

Esta hipótesis es coincidente con la hipótesis 2 de combinación cuasipermanente para el caso de una sobrecarga de uso de valor $3 \mathrm{kN} / \mathrm{m}^{2}$, dado que para dicha valor de carga la normativa fija $\Psi_{2}=0,3$. Para la sobrecarga de uso de libre concurrencia $\left(5 \mathrm{kN} / \mathrm{m}^{2}\right)$, la normativa fija un valor $\Psi_{2}=$ o,6. Sin embargo, consideramos más adecuado a efectos de

Tabla 2a. Resultados para los tipos de forjado nervado según hipótesis.

\begin{tabular}{|c|c|c|c|}
\hline \multirow{2}{*}{ TIPO } & $L$ & $\omega_{c}$ & $\Omega$ \\
\hline & {$[\mathrm{m}]$} & $\begin{array}{c}{[\mathrm{mm}]} \\
\mathrm{L} / \mathrm{K}\end{array}$ & {$\left[\mathrm{mm}^{2}\right]$} \\
\hline \multirow{2}{*}{ T1 } & \multirow[b]{2}{*}{9} & $-25,82$ & \multirow{2}{*}{742,17} \\
\hline & & $L / 349$ & \\
\hline \multirow{2}{*}{ T2 } & \multirow{2}{*}{12} & $-34,83$ & \multirow{2}{*}{1060,83} \\
\hline & & $L / 345$ & \\
\hline \multirow{2}{*}{ T3 } & \multirow{2}{*}{15} & $-45,11$ & \multirow{2}{*}{1419,55} \\
\hline & & $L / 333$ & \\
\hline \multirow{2}{*}{$\mathrm{T}_{4}$} & \multirow[b]{2}{*}{9} & $-19,52$ & \multirow{2}{*}{716,78} \\
\hline & & $L / 461$ & \\
\hline \multirow{2}{*}{$\mathrm{T}_{5}$} & \multirow{2}{*}{12} & $-26,87$ & \multirow{2}{*}{$1.030,71$} \\
\hline & & $L / 447$ & \\
\hline \multirow{2}{*}{ T6 } & \multirow{2}{*}{15} & $-35,33$ & \multirow{2}{*}{$1.386,86$} \\
\hline & & $L / 425$ & \\
\hline
\end{tabular}

\begin{tabular}{|c|c|c|c|}
\hline \multicolumn{4}{|c|}{$\begin{array}{l}\text { HIPÓTESIS DISEÑO-HIPÓTESIS D } \\
\qquad q_{D}=G_{k}+0,3 \cdot Q_{k}\end{array}$} \\
\hline$N_{p o s t, D}$ & $\begin{array}{c}M_{\text {post }, D} \\
{\left[\frac{M_{\text {post }, D}}{M_{\text {isost }, D}}\right]}\end{array}$ & $s_{D}$ & $\omega_{D, \text { inst }}$ \\
\hline$[\mathrm{kN}]$ & $\begin{array}{c}{[\mathrm{m} . \mathrm{kN}]} \\
\%\end{array}$ & $\begin{array}{c}{[\mathrm{mm}]} \\
\mathrm{L} / \mathrm{K}\end{array}$ & $\begin{array}{c}{[\mathrm{mm}]} \\
\mathrm{L} / \mathrm{K}\end{array}$ \\
\hline \multirow{2}{*}{37,88} & 500 & 4,50 & $-9,68$ \\
\hline & $25,29 \%$ & $L / 2.000$ & $L / 929$ \\
\hline \multirow{2}{*}{53,64} & , 14 & 60 & $-13,06$ \\
\hline & $27,21 \%$ & $L / 2.000$ & /919 \\
\hline \multirow{2}{*}{71,10} & $-17,12$ & 7,50 & $-16,91$ \\
\hline & $28,26 \%$ & $L / 2.000$ & $L / 887$ \\
\hline \multirow{2}{*}{36,58} & $-5,27$ & 4,50 & $-7,32$ \\
\hline & $27,09 \%$ & $L / 2.000$ & $L / 1.230$ \\
\hline \multirow{2}{*}{52,11} & 0,4 & 6,00 & $-10,08$ \\
\hline & $28,72 \%$ & $L / 2.000$ & $L / 1.191$ \\
\hline \multirow{2}{*}{69,46} & $-17,56$ & 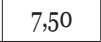 & $-13,25$ \\
\hline & $29,67 \%$ & $L / 2.000$ & $L / 1.132$ \\
\hline
\end{tabular}

\begin{tabular}{|c|c|c|c|}
\hline \multicolumn{4}{|c|}{$\begin{array}{c}\text { HIPÓTESIS } 1 \\
q_{1}=G_{k}\end{array}$} \\
\hline \multirow[t]{2}{*}{$N_{\text {post }, 1}$} & \multirow{2}{*}{$\begin{array}{c}M_{\text {post }, 1} \\
{\left[\frac{M_{\text {post }, 1}}{M_{\text {iost }, 1}}\right.}\end{array}$} & \multirow[t]{2}{*}{$s_{1}$} & \multirow[t]{2}{*}{$\omega_{1, i n s t}$} \\
\hline & & & \\
\hline \multirow[t]{2}{*}[\mathrm{kN}]{} & {$[\mathrm{mkN}]$} & {$[\mathrm{mm}]$} & {$[\mathrm{mm}]$} \\
\hline & $\%$ & $\mathrm{~L} / \mathrm{K}$ & $\mathrm{L} / \mathrm{K}$ \\
\hline \multirow{2}{*}{23,64} & $-3,18$ & 2,78 & $-15,43$ \\
\hline & $24,74 \%$ & $L / 3.240$ & $L / 583$ \\
\hline \multirow{2}{*}{33,99} & $-6,43$ & 3,75 & $-20,44$ \\
\hline & $26,44 \%$ & $L / 3.201$ & $L / 587$ \\
\hline \multirow{2}{*}{45,66} & $-11,00$ & 4,73 & $-26,00$ \\
\hline & $27,27 \%$ & $L / 3.169$ & $L / 577$ \\
\hline \multirow{2}{*}{18,90} & $-2,72$ & 2,29 & $-12,95$ \\
\hline & $26,34 \%$ & $L / 3.926$ & $L / 695$ \\
\hline \multirow{2}{*}{27,77} & $-5,54$ & 3,14 & $-17,42$ \\
\hline & $27,69 \%$ & $L / 3.822$ & $L / 689$ \\
\hline \multirow[t]{2}{*}{38,00} & $-9,61$ & 4,01 & $-22,39$ \\
\hline & $28,37 \%$ & $L / 3.737$ & $L / 670$ \\
\hline
\end{tabular}

\begin{tabular}{|c|c|c|c|c|}
\hline \multicolumn{5}{|c|}{$\begin{array}{c}\text { HIPÓTESIS } 2 \\
q_{2}=G_{k}+\psi_{2} \cdot Q_{k}\end{array}$} \\
\hline$N_{p o s t, 2}$ & \multirow{2}{*}{$\begin{array}{c}M_{\text {post }, 2} \\
{\left[\frac{M_{p o s t, 2}}{M_{i s s s t, 2}}\right]}\end{array}$} & \multirow[t]{2}{*}{$s$} & \multirow[t]{2}{*}{$\omega_{2, \text { inst }}$} & \multirow[t]{2}{*}{$\omega_{2, f i n}$} \\
\hline & & & & \\
\hline$[\mathrm{kN}]$ & {$[\mathrm{m} . \mathrm{kN}]$} & [mm] & [mm] & {$[\mathrm{mm}]$} \\
\hline \multirow{3}{*}{37,88} & & & & \\
\hline & $-5,09$ & 4,50 & $-9,68$ & 0 \\
\hline & $25,29 \%$ & $L / 2.000$ & $L / 929$ & - \\
\hline \multirow{2}{*}{53,64} & $-10,14$ & 6,00 & $-13,06$ & 0 \\
\hline & $27,21 \%$ & $L / 2.000$ & $L / 919$ & - \\
\hline \multirow{2}{*}{71,10} & $-17,12$ & 7,50 & $-16,91$ & 0 \\
\hline & $28,26 \%$ & $L / 2.000$ & $L / 887$ & - \\
\hline \multirow{2}{*}{55,43} & $-7,98$ & 6,92 & $-1,88$ & 8,71 \\
\hline & $27,95 \%$ & $L / 1.301$ & $L / 4.787$ & $L / 1.033$ \\
\hline \multirow{2}{*}{78,79} & $-15,73$ & 9,26 & 3,11 & 11,14 \\
\hline & $29,96 \%$ & $L / 1.296$ & $L / 3.859$ & $L / 1.078$ \\
\hline \multirow[t]{2}{*}{104,73} & $-26,47$ & 11,61 & $-4,79$ & 13,56 \\
\hline & $31,31 \%$ & $L / 1.292$ & $L / 3.132$ & $L / 1.106$ \\
\hline
\end{tabular}

Tabla 2b. Resultados para los tipos de forjado nervado según hipótesis.

\begin{tabular}{|c|c|c|c|c|c|c|c|c|c|c|c|}
\hline \multirow{4}{*}{ TIPO } & \multirow{4}{*}{$\begin{array}{c}L \\
{[\mathrm{~m}]}\end{array}$} & \multirow{4}{*}{$\frac{\omega_{c}}{[\mathrm{~mm}]}$} & \multirow{4}{*}{$\begin{array}{c}\Omega \\
{\left[\mathrm{mm}^{2}\right]}\end{array}$} & \multicolumn{5}{|c|}{$\begin{array}{c}\text { HIPÓTESIS } 3 \\
q_{3}=G_{k}+Q_{k}\end{array}$} & \multicolumn{3}{|c|}{$\begin{array}{c}\text { COMPROBACIONES } \\
\text { NORMATIVAS }\end{array}$} \\
\hline & & & & $N_{\text {post, } 3}$ & $\begin{array}{c}M_{\text {post }, 3} \\
{\left[\frac{M_{\text {post }, 3}}{M_{\text {isost }, 3}}\right]}\end{array}$ & $s_{3}$ & $\omega_{3, \text { inst }}$ & $\omega_{3, f i n}$ & $\omega_{i n t}$ & $\omega_{\text {confort }}$ & $\omega_{\text {apar }}$ \\
\hline & & & & C.NT & {$[\mathrm{m}, \mathrm{kN}]$} & {$[\mathrm{mm}]$} & {$[\mathrm{mm}]$} & {$[\mathrm{mm}]$} & {$[\mathrm{mm}]$} & {$[\mathrm{mm}]$} & {$[\mathrm{mm}]$} \\
\hline & & & & {$[\mathrm{KN}]$} & $\%$ & $\mathrm{~L} / \mathrm{K}$ & $\mathrm{L} / \mathrm{K}$ & $\mathrm{L} / \mathrm{K}$ & $\mathrm{L} / \mathrm{K}$ & $\mathrm{L} / \mathrm{K}$ & $\mathrm{L} / \mathrm{K}$ \\
\hline \multirow{2}{*}{ T1 } & \multirow{2}{*}{9} & $-25,82$ & \multirow{2}{*}{742,17} & \multirow{2}{*}{74,16} & $-9,97$ & 9,07 & 3,16 & 12,85 & 28,28 & 12,85 & 0 \\
\hline & & $L / 349$ & & & $26,84 \%$ & $L / 992$ & $L / 2.846$ & $L / 701$ & $L / 318$ & $L / 701$ & - \\
\hline \multirow{2}{*}{ T2 } & \multirow{2}{*}{12} & $-34,83$ & \multirow{2}{*}{$1.060,83$} & \multirow{2}{*}{105,55} & $-19,96$ & 12,29 & 2,95 & 16,01 & 36,44 & 16,01 & 0 \\
\hline & & $L / 345$ & & & $29,57 \%$ & $L / 977$ & $L / 4.073$ & $L / 750$ & $L / 329$ & $L / 750$ & - \\
\hline \multirow{2}{*}{$\mathrm{T} 3$} & \multirow{2}{*}{15} & $-45,11$ & \multirow{2}{*}{$1.419,55$} & \multirow{2}{*}{141,28} & $-34,02$ & 15,70 & 2,08 & 18,99 & 45,00 & 18,99 & 0 \\
\hline & & $L / 333$ & & & $31,55 \%$ & $L / 955$ & $L / 7.211$ & $L / 790$ & $L / 333$ & $L / 790$ & - \\
\hline \multirow{2}{*}{$\mathrm{T}_{4}$} & \multirow{2}{*}{9} & $-19,52$ & \multirow{2}{*}{716,78} & \multirow{2}{*}{82,95} & $-11,94$ & 10,60 & 5,00 & 15,59 & 28,54 & 12,32 & 8,71 \\
\hline & & $L / 461$ & & & $29,34 \%$ & $L / 849$ & $L / 1.799$ & $L / 577$ & $L / 315$ & $L / 730$ & $L / 1.033$ \\
\hline \multirow{2}{*}{$\mathrm{T}_{5}$} & \multirow{2}{*}{12} & $-26,87$ & \multirow{2}{*}{$1.030,71$} & \multirow{2}{*}{119,22} & $-23,80$ & 14,49 & 5,30 & 19,55 & 36,97 & 15,38 & 11,14 \\
\hline & & $L / 447$ & & & $32,15 \%$ & $L / 828$ & $L / 2.265$ & $L / 614$ & $L / 325$ & $L / 780$ & $L / 1.078$ \\
\hline \multirow{2}{*}{ T6 } & \multirow{2}{*}{15} & $-35,33$ & \multirow{2}{*}{$1.386,86$} & \multirow{2}{*}{161,72} & $-40,88$ & 18,77 & 4,76 & 23,11 & 45,50 & 18,01 & 13,56 \\
\hline & & $L / 425$ & & & $34,57 \%$ & $L / 799$ & $\mathrm{~L} / 3152$ & $L / 649$ & $L / 330$ & $L / 833$ & $L / 1.106$ \\
\hline
\end{tabular}


diseño mantener distorsión nula para una fracción menor de la sobrecarga de uso.

En esta hipótesis se fija el asiento de diseño $\left(s_{D}\right)$ a partir del cual se determina el área del tendón y el valor de la contraflecha $\left(\omega_{c}\right)$ con el que se asegura que la flecha final (instantánea + diferida) relativa o distorsión es nula para esta hipótesis. En todos los casos analizados se ha considerado un asiento de diseño $s_{D}=L / 2.000$.

\section{- Hipótesis 1}

Situación en la que sobre el sistema sólo actúa la acción $\left(q_{1}\right)$ debida al peso propio y la carga permanente.

$$
q_{1}=\sum_{j \geq 1} G_{k, j}
$$
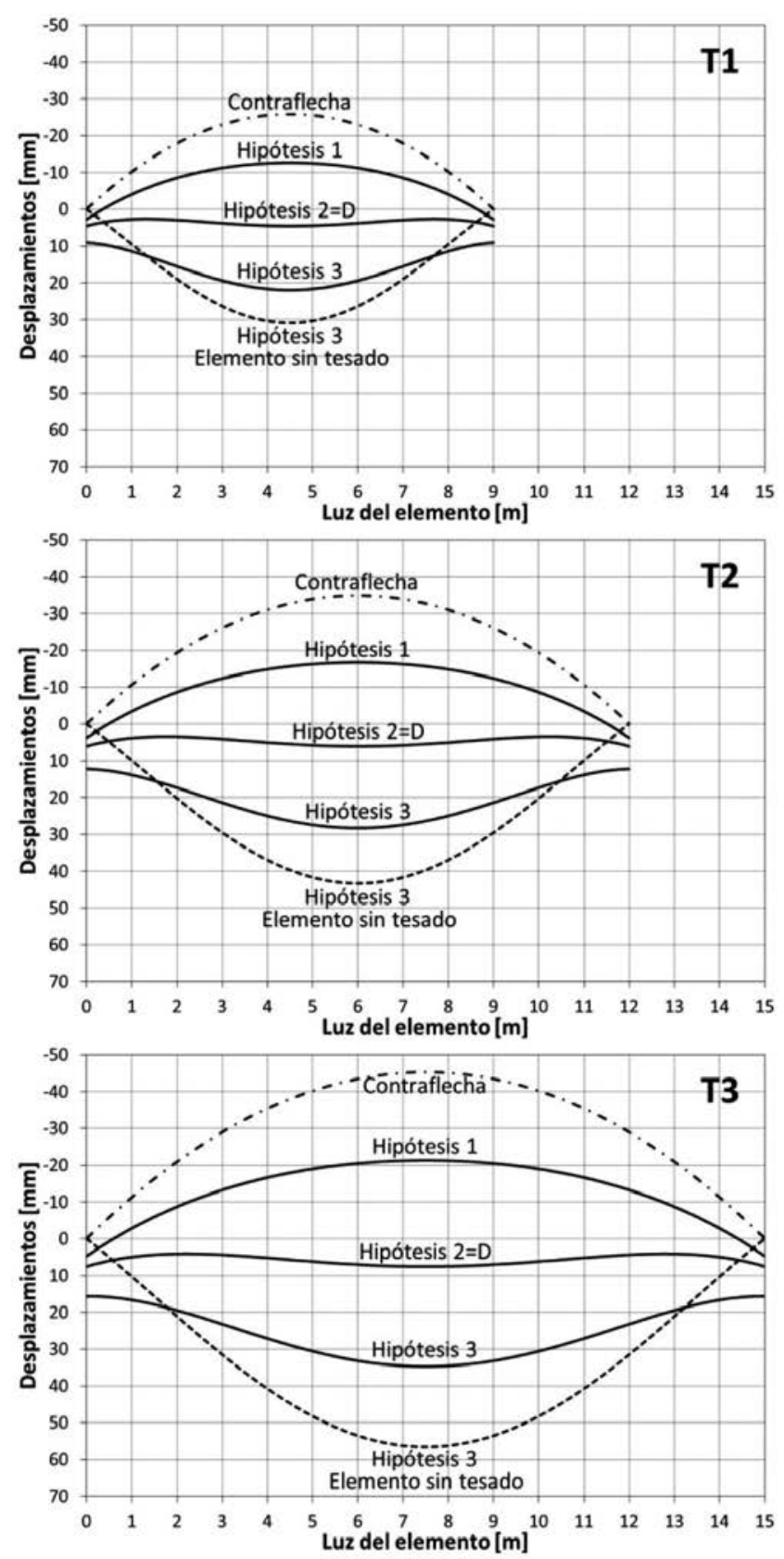

- Hipótesis 2

Esta segunda hipótesis corresponde a la actuación de una acción exterior $\left(q_{2}\right)$ en combinación cuasipermanente:

$$
q_{2}=\sum_{j \geq 1} G_{k, j}+\sum_{i \geq 1} \psi_{2, i} \cdot Q_{k, i}
$$

- Hipótesis 3

Corresponde a la actuación de una acción exterior en combinación característica de carga total $\left(q_{3}\right)$.

$$
q_{3}=\sum_{j \geq 1} G_{k, j}+Q_{k, 1}+\sum_{i \geq 1} \psi_{0, i} \cdot Q_{k, i}
$$

Los resultados obtenidos se recogen en la Tablas $2 a$ y $2 b$, en las que figuran las distintas hipótesis contempladas, así como en las gráficas de la Figura 4. Se puede apreciar la influencia
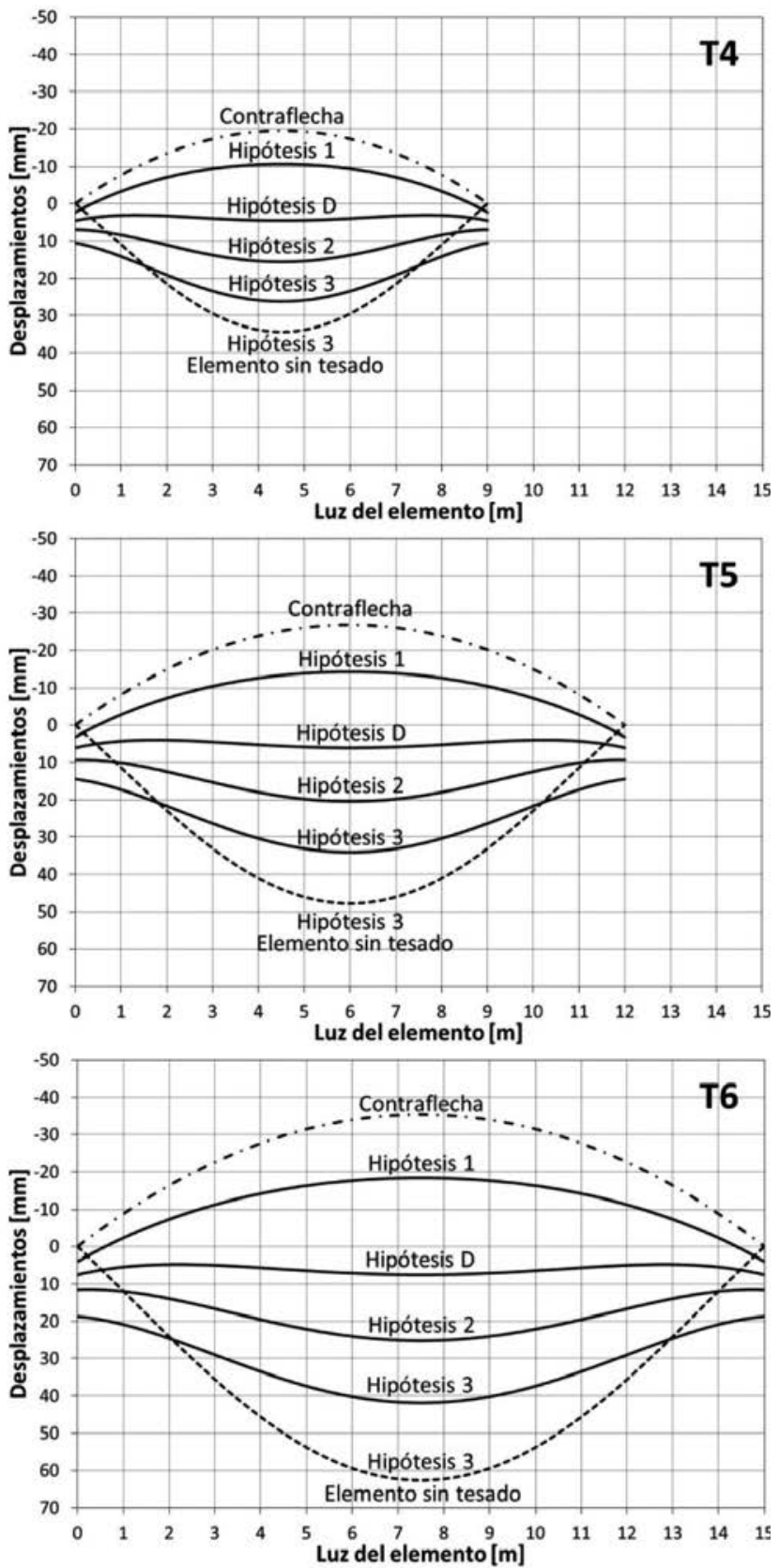

Figura 4. Deformadas comparativas de las secciones analizadas. 
que tiene el comportamiento no lineal del dispositivo multiplicador en el comportamiento del sistema. A medida que se incrementa la magnitud de la acción aumenta el efecto multiplicador y, por tanto, su efectividad. Este efecto se puede comprobar observando cómo el momento generado por el postesado representa un porcentaje del momento isostático que se incrementa con la magnitud de la acción actuante.

Así, en el caso de la sección T1, el porcentaje pasa de 24,74 \% en la hipótesis 1 a 25,29 \% en la hipótesis 2, hasta alcanzar el 26,84 \% en la hipótesis 3. Si cogemos la sección tipo T6 estos porcentajes son, respectivamente, de 28,37 \%, 31,31 \% y 34,57\%.

La eficacia del sistema de autotesado se aprecia con claridad en la Tabla $2 b$ que recoge la verificación de las exigencias de deformación en los tres casos contemplados por la normativa: integridad de elementos constructivos $\left(\omega_{\text {int }}\right)$, confort de usuarios $\left(\omega_{\text {confort }}\right)$ y apariencia $\left(\omega_{\text {apar }}\right)$. Se puede observar que con una elección adecuada de los parámetros de diseño (proceso de enorme sencillez con la metodología de análisis expuesta) podemos diseñar forjados nervados de elevada luz y carga variable de uso cuya deformación relativa o distorsión por integridad de elementos constructivos (la exigencia más desfavorable) satisface en todos los casos el valor $L / 300$.

La exigencia de flecha relativa por confort de usuarios, que la normativa limita a $L / 350$, alcanza en todas las secciones valores inferiores a $L / 700$.

Finalmente en relación con la flecha relativa por apariencia, en el caso de las secciones $\mathrm{T} 1$ a T3 el valor es nulo por cuanto corresponde a la hipótesis establecida por diseño para la obtención de distorsión nula. En el caso de las secciones T4 a T6 los valores son todos inferiores a $L / 1$.00o frente al valor límite establecido por la normativa de $L / 300$.

En consecuencia, se observa que el sistema de autotesado, con una fuerza que varía con la magnitud de las cargas ac- tuantes, permite diseñar forjados de elevada luz con una gran esbeltez con una distorsión nula o casi nula en la situación de servicio de la estructura.

En este sentido las gráficas recogidas en la Figura 4 son elocuentes. Podemos observar como para las tres luces analizadas $(9,12$ y $15 \mathrm{~m})$ y considerando dos valores de carga variables ( 3 y $5 \mathrm{kN} / \mathrm{m}^{2}$ ), en todos los casos la distorsión para la hipótesis cuasipermanente, que corresponde a la situación habitual de servicio, es nula (casos T1 a T3) o de magnitud muy reducida ( $\mathrm{T} 4 \mathrm{a}$ T6).

\section{CONCLUSIONES}

El sistema de autotesado consistente en la colocación en los apoyos de un dispositivo multiplicador de fuerza conectado a un tendón de tesado constituye un sistema de elevada eficiencia que permite diseñar forjados nervados de madera de elevada luz empleando cantos reducidos. La eficacia del sistema deriva fundamentalmente de una fuerza de tesado cuya magnitud varía con la de las cargas actuantes.

La complejidad de cálculo debido a la no linealidad del sistema se puede obviar con la metodología de análisis desarrollada en el artículo. El establecimiento de un asiento de diseño para una determinada hipótesis combinatoria de carga permite obtener con gran sencillez todos los parámetros determinantes del sistema que aseguran un comportamiento eficiente del conjunto.

\section{AGRADECIMIENTOS}

Esta investigación forma parte del proyecto «Sistemas prefabricados de altas prestaciones en madera laminada pretensada con tendones no adherentes» financiado por el Ministerio de Economía y Competitividad y el Fondo Europeo de Desarrollo Regional (FEDER).

\section{REFERENCIAS}

(1) Estévez Cimadevila, J., Otero Chans, D., Martín Gutiérrez, E., Suárez-Riestra, F. (2016). Self-tensioning system for longspan wooden structural floors. Construction and Building Materials, 102, Part 1: 855, doi: http://dx.doi.org/10.1016/j. conbuildmat.2015.11.024.

(2) Otero Chans, D., Estévez Cimadevila, J., Martín Gutiérrez, E., Pérez Valcárcel, J. (2017). Application of a new system of self-tennsioning to the design of large-span wood floor framings. Journal of Structural Engineering, 142(6), doi: http:// dx.doi.org/10.1061/(ASCE)ST.1943-541X.0001486.

(3) Celigüeta, J. T. (2009). Análisis de estructuras con no linealidad geométrica, p. 153. Ed. Tecnum, Universidad de Navarra.

(4) Deuflhard, P. (2004). Newton Methods for Nonlinear Problems (Affine invariance and Adaptative Algorithms). Springer Series in Computational Mathematics.

(5) UNE-EN 1990:2003/A1:2010 Eurocode o: Basis of Structural Design.

(6) European Committee for Standardization (CEN). Timber structures. Glued laminated timber and glued solid timber. Requirements. EN 14080:2013.

(7) European Committee for Standardization (CEN). Timber structures. Cross laminated timber. Requirements. EN 16351:2016. 